\title{
Invited Talk II Summary: Incentive Schemes in Memory-Less P2P Systems
}

\author{
Costas Courcoubetis \\ Department of Computer Science, \\ Athens University of Economics and Business, \\ 76 Patision str., Athens, GR 10434 \\ courcou@aueb.gr
}

The asymptotic analysis of certain public good models for $\mathrm{p} 2 \mathrm{p}$ file sharing systems focusing on content availability, suggests that when the aim is to maximize social welfare, a fixed contribution scheme in terms of the number of files shared per unity of time can be asymptotically optimal as the number of participants $n$ grows to infinity (see [1] and references therein). Such an incentive scheme is very simple and attractive, and is also suitable for other $\mathrm{p} 2 \mathrm{p}$ applications with similar public good charasteristics such as WLAN peering. However, its enforcement is not straightforward in cases where no trusted software or central entity accounting for peers' transactions can be assumed and peers are free to change their identity with no cost. That is, when no sort of user memory is available to be able to identify and punish the potential free riders.

A 'memory-less' p2p system should rely only on the time peers are consuming resources to ensure that they contribute adequately. BitTorrent is an example of a successful real world application focusing on bandwidth provisioning for content distribution, which implements a reciprocative incentive scheme without relying on past transactions of peers but on a direct exchange of resources (i.e. upload bandwidth). BitTorrent, however, does not tackle the objective of improving content availability.

Recent articles in the popular press discuss the importance of the 'long tail' of content; that large part of the set of content in which individual files are not popular, but which together constitute the majority of the total requests. The provision of this part of the content in a p2p system requires different types of incentives than the ones usually discussed in the $\mathrm{p} 2 \mathrm{p}$ economics literature, which look at uploading cost rather than the cost for contributing to the overall content availability.

We therefore choose to consider a system where the probability of a certain file being requested is low but the overall value of satisfying such requests is much greater than for popular items (since it is more difficult to find unpopular or rare items, in many cases even if one wishes to pay for them). Thus we will attempt to give incentives for providing any content item regardless of request rate, considering uploading cost to be of limited importance, especially since we require that this cost is incurred only while peers are consuming resources. But

The original version of this chapter was revised: The copyright line was incorrect. This has been corrected. The Erratum to this chapter is available at DOI: 10.1007/978-3-540-32993-0_29

* Supported in part by the IST FET Coordination Action ACCA (IST-6475).

I. Stavrakakis and M. Smirnov (Eds.): WAC 2005, LNCS 3854, pp. 281-282, 2006.

(C) Springer-Verlag Berlin Heidelberg 2006 
we also need to give incentives to remain in the system and providing content, not simply to have provided at some time in the past.

We thus propose the following "contribute while consuming" incentive mechanism enforced by uploading peers, which

1. check that the downloaders share a predefined number of valid files

2. use a certain (not too low) upload throughtput in order to ensure that these files are made available for a significant amount of time.

Of course, there are some important implementation and incentive issues that arise in this context (e.g. ensuring the validity of files shared, the need for super peers in order to avoid the requirement of cycles of requests to be formed, and more), which are discussed in detail in [2]. In any case, we believe that time spent in $\mathrm{p} 2 \mathrm{p}$ systems will become a critical parameter of the contribution of the participating peers, and especially in the case of file sharing as access speeds increase and people store more content in their PCs for their own use.

So, in [2] we made a first effort to formulate a corresponding economic model in order to provide a sound theoretical framework for the study of the qualitative characteristics of our memory-less incentive mechanism and provide insights for the appropriate tuning of its basic parameters (the number of files shared and the upload throughput used by all peers in the system). Our results show that the resulting efficiency is comparable to the one achieved using the theoretically optimal schemes, which is very encouraging taking into account the very limited implementation requirements of this mechanism.

Our on-going work includes the in-depth analysis of the proposed economic model (using both simulations and analytical tools). We also wish to explore to what extend our public good model and/or memory-less enforcement mechanism are applicable in other p2p systems with public good characteristics.

\section{References}

1. Courcoubetis, C., Weber, R.R.: Incentives for large p2p systems. Accepted for publication in IEEE Journal on Selected Areas in Telecommunications, available at http://nes.aueb.gr/p2p.html (2005)

2. Antoniadis, P., Courcoubetis, C., Strulo, B.: Incentives for Content Availability in Memory-less Peer-to-Peer File Sharing Systems. ACM SIGecom Exchanges 5(4) (2005) 11-20 